\title{
ANÁLISE DE MARCADORES TUMORAIS NO MUNICÍPIO DE SERRINHA-BA/BRASIL
}

\author{
Ivaneide de Jesus Teixeira Silva'; Elielson Souza Rodrigues ${ }^{2, A}$; José Lázaro L. Ribas; \\ Antônio de O. Costa Neto ${ }^{3}$
}

${ }^{1}$ Especialista em Biologia Celular/UEFS: Universidade Estadual de Feira de Santana - Ba/Brasil.

${ }^{2}$ Prof. Dr. Orientador e Coordenador do Laboratório Biologia Celular UEFS: Universidade Estadual de Feira de Santana - Ba/Brasil.

${ }^{3}$ Prof. Dept. Ciências Biológicas UEFS: Universidade Estadual de Feira de Santana - Ba/Brasil.

\section{RESUMO}

O câncer é uma das causas de maior mortalidade e morbidade em todo o mundo, sendo caracterizado como um dos principais problemas de saúde pública. Por isso existe uma necessidade premente em identificar marcadores tumorais específicos para auxiliar no diagnóstico e evitar a recidiva do câncer. Estes marcadores tumorais são componentes celulares, estruturais e bioquímicos que conseguem definir alterações celulares e moleculares associadas à conversão de malignidade. Idealmente, um marcador tumoral deveria elevar-se no sangue somente quando ocorresse a presença de tumores e não quando o individuo apresentasse alguma outra doença. O valor clínico para qualquer marcador tumoral sempre dependerá de sua sensibilidade, especificidade, bem como de sua aplicação clínica. Marcadores tumorais conhecidos atualmente não são suficientemente sensíveis para serem utilizados na triagem populacional ou para diagnostico primário de câncer porque nem todos os tumores são produtores eficientes de marcadores. O objetivo desse trabalho é analisar as tendências das alterações nos valores séricos dos marcadores tumorais biológicos (AFP, AC 19.9, CEA) em 200 indivíduos usuários do Sistema Único de Saúde (SUS), que frequentam a unidade laboratorial de saúde pública (Laboratório Municipal de Referência Regional/Lacen Serrinha) do município de Serrinha-BA com idade entre 30 e 70 anos e que residam na mesma cidade. O marcador tumoral AFP e o CA19.9, foram os que apresentaram maior percentual de elevação na população em estudo, estando em conformidade com literatura consultada.

Palavras-chave: Antígenos carcinoembrionário; Alfafetoproteína (AFP); Antígenos carboidratado (CA 19.9).

\section{ABSTRACT}

Cancer is a disease that has increased quite considerably around the world, mainly from the last century. Thus, the use of tumor markers is helping more and more as an aid to diagnosis, as well as cancer relapsed and other factors. The tumor markers, however, are cellular, structural and biochemical components that may define the cellular and molecular changes in both normal cells and those associated with malignant transformation. Ideally, a tumor marker should rise in the blood occur only in presence of tumors and not when the individual presents some other disease. The clinical value for any tumor marker will always depend on their sensitivity, specificity, and its clinical application. Hitherto known markers are not sensitive enough in population screening or primary diagnosis of cancer because 
not all tumors are efficient markers producers. However, the aim of this study is to analyze the trends of changes in serum levels of biological tumor markers (AFP, CA 19.9, CEA) in 200 users of the Unified Health System (SUS), who attends the laboratory unit of public health (Laboratory Municipal Regional Reference / Lacen Serrinha of Serrinha-BA municipality aged 30 and 70 and residing in the same city. The tumors marker AFP and CA 19.9 were the ones with the highest percentage of elevation among the study population, which is in accordance with the literature consulted.

Keywords: Carcinoembryonic antigens; Alphafetoprotein (AFP); Carbohydrate antigens (CA19.9).

\section{INTRODUÇÃO}

As pesquisas em biologia molecular trouxeram grandes avanços na compreensão da gênese e progressão de várias doenças, incluindo o câncer e possibilitaram o desenvolvimento de abordagens diagnósticas e terapêuticas baseadas nestes conhecimentos. Nesse sentido os marcadores tumorais biológicos são muito importantes, pois são componentes celulares, estruturais e bioquímicos, que podem definir alterações celulares e moleculares tanto em células normais quanto aquelas associadas à transformação maligna [1]. Essas substâncias funcionam como indicadores da presença de alterações celulares, e podem ser produzidas diretamente pelo tumor ou pelo organismo, em resposta à presença de neoplasias ou outras doenças não malignas. Em sua maioria os marcadores tumorais são proteínas, antígenos de superfície celular, proteínas citoplasmáticas, enzimas ou hormônios [2]. Esses marcadores podem ser úteis no manejo clínico dos pacientes com câncer, auxiliando nos processos de diagnóstico, estadiamento, avaliação de resposta terapêutica, detecção de recidivas e prognóstico, além de auxiliar no desenvolvimento de novas modalidades de tratamento. Podem ser caracterizados ou quantificados por meios bioquímicos ou imunohistoquímicos nos tecidos ou no sangue, e por testes genéticos para pesquisas de oncogenes, genes supressores de tumores e alterações genéticas [3]. Portanto, buscando explorar a utilidade clínica desses exames é que essa pesquisa buscou analisar as tendências das alterações nos valores séricos dos marcadores tumorais biológicos Alfafetoproteína (AFP), Antígeno Carcinoembrionário (CEA), Antígeno Carboidratado (CA 19.9), em 200 usuários do Sistema Único de Saúde (SUS), que frequentam a Unidade Laboratorial de Saúde Pública do Município de SerrinhaBa/Brasil.

\section{REVISÃO BIBLIOGRÁFICA}

Os marcadores tumorais biológicos podem ser de dois tipos: marcadores intermediários, que medem alterações celulares e moleculares antes do aparecimento da malignidade, e marcadores diagnósticos, presentes em associação com a malignidade, por exemplo: o núcleo, a angiogênese, os microvilos e os microácinos que podem ser medidos de forma quantitativa por métodos bioquímicos, imunológicos, morfométricos, ultra estruturais e moleculares nos fluidos ou nos tecidos corporais, associados a neoplasias e possivelmente ao órgão de origem no caso de eventual neoplasia [1]. Nas últimas décadas, várias proteínas e pequenos peptídeos têm sido identificados como produtos de secreção de diferentes neoplasias sólidas, podendo ser utilizados como marcadores tumorais diagnósticos. Em 1848, Bence Jones descreveu a proteína do mieloma múltiplo, primeira substância empregada clinicamente como marcador tumoral, o que marcou a primeira fase da história dos marcadores tumorais. A segunda fase se caracterizou pela aplicação dos hormônios, enzimas, isoenzimas e outras proteínas como auxiliares para o diagnóstico de neoplasias. O início do terceiro período é marcado pelas descobertas da alfafetoproteína e do antígeno carcinoembriônico. Já o quarto período começou em 1975, com o desenvolvimento dos anticorpos monoclonais e sua aplicação na detecção de antígenos específicos derivados de linhagens celulares neoplásicas, como os antígenos CA 125, CA 15-3, CA 549. A partir de 1980, com o advento da biologia molecular, foi possível detectar alterações cromossômicas com uso associado de sondas moleculares e anticorpos monoclonais, o que possibilitou o estudo dos oncogenes e dos genes supressores [4].

Desde estão, várias outras substâncias vêm sendo pesquisadas em neoplasias de diferentes sítios primários, com o objetivo de avaliar a sua aplicação na prática clínica. De Acordo com as recomendações do INCA, a utilização dos marcadores tumorais tem as seguintes finalidades: triagem populacional, diagnóstico diferencial em pacientes sintomáticos, estadiamento clínico, estabelecimento do diagnóstico, monitorização da eficiência terapêutica, localização de metástases, tratamento (imunorradioterapia) e deteç̧ão precoce da recorrência (grande utilidade). Contudo a maioria dos marcadores disponíveis peca pela falta de especificidade e sensibilidade. Na maioria das vezes o marcador está presente tanto em tecidos benignos quanto em malignos sem possuir um valor de corte claro que permita a distinção entre os dois processos e, em outras situações, apresentam alteração significativa em sua concentração, apenas quando o processo de malignidade já se encontra em estado avançado, como ocorre no câncer de ovário. Assim, os marcadores tumorais atuais são mais bem utilizados no monitoramento do tratamento, avaliação de prognóstico e detecção de recidivas de câncer [5]. De acordo com alguns pesquisadores, os exames laboratoriais e de imagem, utilizados na detecção precoce de um determinado tipo de câncer, não fazem o diagnóstico da doença, mas sim selecionam as pessoas com suspeita de ter esse tipo de 
câncer [6], sendo necessários que testes mais específicos sejam realizados e seja confirmada ou afastada tal suspeita. Geralmente, o teste confirmatório é uma biópsia ou análise anatomopatológica de determinado tecido, muitas vezes solicitado e realizado quando a doença já está instalada.

Para que um teste seja considerado de "screening", ou seja, para que seja considerado adequado para ser realizado em uma pessoa que não apresente sinais e sintomas da doença, ele deve: Ter a capacidade de diagnosticar o câncer, antes que a pessoa desenvolva sintomas; Oferecer pouco risco ou desconforto para a pessoa; Ter um custo acessível.

Por outro lado, desenvolver métodos de detecção precoce em relação a um determinado tipo de câncer, é necessário: Haver evidências científicas suficientes de que, se esse câncer for diagnosticado precocemente, haverá um tratamento médico disponível que reduza a evolução da doença; Que esse tratamento não seja pior que a própria doença.

Além dessas características descritas anteriormente, a detecção precoce deve ser realizada só naqueles cânceres que, se diagnosticados precocemente e instituído tratamento efetivo precocemente, podem modificar o custo afetivo, pessoal e financeiro relacionado ao diagnóstico e ao tratamento ou diminuir a mortalidade relacionada com esse tipo de câncer. Ou seja, não adianta fazer diagnóstico precoce e tratar o câncer na sua fase pré-clínica, se isto não vai alterar a mortalidade ou o sofrimento da pessoa porque, nesse caso, há o risco de tornar uma pessoa saudável e assintomática em uma pessoa doente com todos os custos pessoais e financeiros relacionados ao diagnóstico e ao tratamento de uma doença como o câncer [7]. O câncer é uma patologia com localizações e aspectos clínico-patológicos múltiplos e não possui sintomas ou sinais patognomônicos, podendo ser detectado em vários estágios de evolução histopatológica e clínica. Destes fatos resulta, em grande parte, a dificuldade do seu diagnóstico e a afirmativa de que a suspeita de câncer pode surgir diante dos sintomas mais variados possíveis. Com o avanço da ciência e da tecnologia e o advento da biologia molecular, tem sido possível estudar e identificar os vários mecanismo da carcinogênese, e os principais fatores para sua causa, como a exposição dos seres humanos a determinadas substâncias presente no meio ambiente ou no seu local de trabalho que podem levar ao desenvolvimento de câncer. Geralmente decorre um longo período, podendo levar vários anos entre os eventos iniciais (exposição do carcinógeno, ocorrência da lesão no DNA e fixação das mutações) e o surgimento dos tumores [8].

Como o desenvolvimento de um tumor requer muitos eventos que ocorrem durante longo período de tempo, a indução do câncer em humanos muitas vezes está dentro de um contexto que envolve o ambiente e as substâncias químicas nele presente, aos quais as pessoas estão expostas ao longo da vida. O individuo, ao procurar um médico, não sabe ainda à natureza da sua doença e, assim, não procura diretamente um especialista. Setenta por cento dos diagnósticos de câncer são feitos por médicos não-cancerologistas, o que evidencia a importância destes "profissionais no controle da doença" [9]. O Brasil e diversos países da América Latina estão experimentando, nos últimos 20 anos, uma rápida transição demográfica, epidemiológica e nutricional. A mudança na estrutura etária da população brasileira iniciouse na década de 40 , mas esta tendência acentuou-se nos anos 70 , quando a participação dos indivíduos com 60 anos ou mais passou de $5 \%$ para 6,1\% em 1980, chegando a 7,7\% em 1991 . Em 2000, 15,5 milhões de habitantes possuíam 60 anos ou mais, representando cerca de $10 \%$ da população geral [10]. Associada ao envelhecimento da população, o processo de urbanização e as mudanças socioeconômicas, alimentares e no estilo de vida, houve uma consequente modificação no perfil morbimortalidade nos últimos 20 anos, caracterizando a transição epidemiológica no Brasil [11].

O câncer é uma doença genética em que todos os tumores malignos mantêm um ponto comum: a perda do controle da divisão celular. No entanto, o seu estudo pode também expressar as condições de vida de fatores de risco que são fundamentais para o desenvolvimento da maioria dos tipos câncer [12]. Diante das mudanças no perfil demográfico da população mundial e mais especificamente no Brasil, as doenças crônicas, degenerativas, especialmente as cardiovasculares e o câncer, tem aumentado consideravelmente, por isso diversos estudos têm sido realizados com o intuito de compreender os mecanismos patológicos e desenvolver a melhor forma de prevenir, diagnosticar e tratar a doença. Dentre os marcadores tumorais apresentados, o CA 19.9 (antígeno carboidrato), o AFP (alfafetoproteínas) e o CEA (antígeno carcinoembrionário), foram os selecionados para ser analisado e quantificado no estudo proposto, por apresentar sensibilidade e especificidade frente a patologias na região pancreática, hepática e gastrointestinal pois, de acordo com pesquisa realizadas pelo projeto Globocan, a incidência de neoplasias nesses tecidos tem aumentado mundialmente a cada ano e as causas do seu aumento estão relacionadas com o novo estilo de vida e características da população, uma vez que o câncer de pâncreas, por exemplo, torna-se mais comum a partir dos 60 anos e que tende a aumentar de acordo com a idade. Em pessoas com idade entre 40 e 50 anos esta estimativa é de 10/100.000 habitantes. Já na população mais idosa, entre 80 e 85 anos, a estimativa é de 116/100.000 habitantes, além de apresentar uma incidência mais significativa em homens do que em mulheres, devido a aqueles apresentarem uma maior exposição aos agentes carcinógenos [9].

O câncer de pâncreas mais comuns é do tipo adenocarcinoma (que se origina no tecido glandular), correspondendo a $90 \%$ dos casos diagnosticados. A maioria dos casos afeta o lado direito do órgão (a cabeça). Pelo fato de ser de difícil detecção, o câncer de pâncreas apresenta alta taxa de mortalidade, por conta do diagnóstico tardio e de seu comportamento agressivo. Segundo o Ministério da Saúde, em 2015 foi estimado que este tipo de câncer foi responsável por cerca de $2 \%$ de todos os tipos de câncer diagnosticados e por $4 \%$ do total de mortes por essa doença, sendo 8.710 o número de óbitos por ano [11]. Os tumores malignos de fígado podem ser divididos em dois tipos: câncer primário (que tem sua origem no próprio órgão) e secundário ou metastático (originado em outro órgão e que atinge também o fígado). 
Dentre os tumores originados no fígado, o mais frequente é o hepatocarcinoma ou carcinoma hepatocelular. Agressivo, ocorre em mais de $80 \%$ dos casos. Outros tipos de câncer primário de fígado são o colangiocarcinoma (originado nos dutos biliares do fígado), o angiossarcoma (câncer raro que se origina nos vasos sanguíneos do órgão) e o hepatoblastoma, tumor maligno raro que atinge recém-nascido e crianças nos primeiros anos de vida. O Ministério da Saúde estimou, em 2015, que o câncer hepático foi responsável pelo total de 8.772 óbitos por ano no Brasil [11]. As características dos marcadores tumorais estão relacionadas com os tipos específicos de tecido onde são expressos ou produzidos. Assim os marcadores tumorais biológicos selecionados na pesquisa, apresentam-se com antígenos de superfície celular que sinalizam as alterações nos tecidos hepáticos, pancreáticos e gastrointestinais:

Antígeno Carcinoembrionário (CEA) - é o protótipo marcador tumoral que tem sido extensivamente estudado desde sua identificação, em 1965. Originalmente, foi descrito como presente em Adenocarcinoma de cólon e reto e em cólon fetal, mas ausentes em tecido colônico adulto normal. Atualmente, sabese que CEA é produzido pelas células da mucosa gastrintestinal, tem peso molecular de aproximadamente $200 \mathrm{Kd}$ e faz parte da família das imunoglobulinas. Seu valor de referência é $3,5 \mathrm{ng} / \mathrm{mL}$ em não fumantes e $7 \mathrm{ng} / \mathrm{mL}$ em fumantes. Na neoplasia maligna, níveis elevados de CEA são detectados em $9 \%$ dos teratomas de testículos, e em aproximadamente $85 \%$ dos casos de carcinoma colorretal metástico. Níveis elevados de CEA também são encontrados em outras neoplasias malignas, como por exemplo, pulmão $(52 \%$ a $77 \%)$, pâncreas $(61 \%$ a $68 \%)$, trato gastrintestinal ( $40 \%$ a $60 \%)$, trato biliar $(80 \%)$, tireóide $(50 \%$ a $70 \%)$, cérvice $(42 \%$ a $50 \%$ ) e mama ( $30 \%$ a $50 \%$ ). A sensibilidade do CEA oscila em torno de $40 \%$ a $47 \%$ e a especificidade, $90 \%$ a $95 \%$ para câncer de colorretal, e $95 \%$ a $100 \%$ para câncer recorrente. Elevações do CEA também foram relatados em distúrbios benignos, como cirrose alcoólica, doença de Crohn, doença hepática, doença intestinais, doença fibrocística de mama, bronquite, tabagismos e insuficiência renal. Por conseguinte, os ensaios do CEA carecem de especificidade e de sensibilidade necessárias para a detecção de câncer no estágio inicial [18; 19].

Alfa Feto Proteína (AFP) - É uma importante proteína do soro fetal, que é sintetizada no fígado, saco vitelino e intestino do feto, com função de transporte plasmático e de manutenção da pressão oncótica, desaparecendo no primeiro ano de vida. $\mathrm{Na}$ vida adulta seus níveis séricos encontram-se entre $5 \mathrm{ng} / \mathrm{mL}$ e $15 \mathrm{ng} /$ $\mathrm{mL}$ possui vida média de 5-7 dias. Níveis acima de $500 \mathrm{ng} / \mathrm{mL}$ são altamente sugestivos de malignidade e valores acima de 1000ng/ $\mathrm{mL}$ são indicativos de presença de neoplasias. Essa proteína pode está elevada em pacientes portadores de tumores gastrintestinais, hepatite, cirrose, hepatocarcinoma e gestantes, podendo ser encontrada em $70 \%$ dos tumores testiculares não seminomatosos. Esse marcador tem como principal papel a monitorização da terapia para o carcinoma de testículo, sendo que sua presença sugere persistência da doença e sua concentração sérica propicia uma estimativa do tempo de crescimento tumoral inicial [20; 21].
Antígenos Carboidratado (CA 19.9) - É um antígeno carboidrato de superfície celular, com peso molecular variando de $200 \mathrm{Kd}$ a $1000 \mathrm{Kd}$, sendo também conhecido como antígeno de Lewis. É liberado na superfície da célula cancerosa e penetra na corrente sanguínea, onde pode ser detectado. Seu valor normal de referência é $37 \mathrm{U} / \mathrm{mL}$. Este marcador tumoral é indicado no auxílio ao estadiamento e à monitoração de tratamento em primeira escolha de câncer de pâncreas e trato biliar e, em segunda escolha, no câncer colorretal. O CA 19.9 possui sensibilidade variável com a localização do tumor: pâncreas $70 \%$ a $94 \%$, vesícula biliar $60 \%$ a $79 \%$, hepatocelular $30 \%$ a $50 \%$, gástrico $40 \%$ a $60 \%$ e colorretal $30 \%$ a $40 \%$. Algumas doenças como cirrose hepática, pancreatite, doença inflamatória intestinal pode elevar o CA 19.9 , sem ultrapassar $120 \mathrm{U} / \mathrm{mL}$. No câncer de pâncreas, o CA 19.9 tem especificidade de $81 \%$ a $94 \%$, sendo utilizado no diagnóstico diferencial de câncer de pâncreas e de pancreatite. Há um aumento de CA 19.9 em cerca de 99\% dos casos de câncer de pâncreas, enquanto nas pancreatites crônicas é $4 \%$ a $10 \%$ e nas pancreatites agudas, $23 \%$. Atualmente, parece ser um dos marcadores mais sensíveis e específicos usados para o diagnóstico diferencial do câncer de pâncreas e de vesícula, apresentando $79,4 \%$ de sensibilidade e $79,2 \%$ de especificidade quando maior do que $20 \mathrm{U} / \mathrm{mL}$. No momento, a maior aplicabilidade de uso do CA 19.9 é a de avaliar resposta à quimioterapia do câncer de pâncreas, já que a utilização de métodos de imagem é bastante limitada para este fim. Cada marcador tumoral apresenta valores séricos específicos que determina o grau de normalidade ou de alterações de acordo com uma faixa de referência estipulada, cada valor estabelecido foi avaliado e estudado observando-se um grau de compatibilidade em população avaliada. Os valores de referência dos marcadores tumorais utilizados na pesquisa podem ser observados abaixo [22; 23].

Tabela 1. Valor de referência dos marcadores tumorais CEA, AFP, CA 19.9 de acordo com a tabela do LACEN/BA.

\begin{tabular}{|c|c|}
\hline MARCADOR TUMORAL & VALOR DE REFERÊNCIA \\
\hline CEA & $\leq 5.0 \mathrm{ng} / \mathrm{Ml}$ \\
\hline AFP & 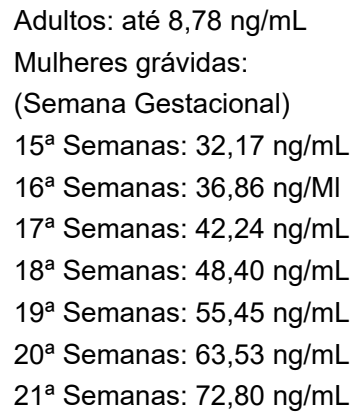 \\
\hline CA 19.9 & $<2,0 \mathrm{U} / \mathrm{mL}$ \\
\hline
\end{tabular}




\section{MATERIAL E MÉTODOS}

A abordagem utilizada neste trabalho se baseia na pesquisa de campo do tipo quantitativa descritiva, a qual segundo Marconi \& Lakatos [13], consiste em investigações de dados obtidos previamente e, que tem como finalidade o delineamento ou análise das características de fatos. Para as análise estatística inferencial dos dados foram feitos gráficos no Excel comparando todas as possibilidades entre as proporções dos marcadores tumorais biológicos, tanto na população de homens como de mulheres e também os dois juntos. Para verificação das diferenças estatísticas, foram feitos testes entre as proporções dos marcadores em condições elevadas, usando o programa Estatística, Versão 5.0 [14], (teste para diferença entre proporções), levando em consideração uma confiança de $95 \%(p<0,05)$.

A metodologia foi submetida à análise e autorização para sua realização pelo Comitê de Ética em Pesquisa com Seres Humanos da Universidade Estadual de Feira de Santana (CEP/UEFS) e a pesquisa e coleta de dados só foi iniciada após a liberação do termo de aceite do CEP, (aprovação do projeto nos Comitês de Ética tem a seguinte numeração CAAE: 50339815.2.0000.0053) iniciou-se a pesquisa a partir de convites feitos diretamente aos usuários da unidade laboratorial de saúde (Laboratório Municipal de Referência Regional - LACEN/Serrinha-Ba). Foram convidados a participar da pesquisa 200 usuários utilizam os serviços de saúde da unidade laboratorial de Análise Clínica, (Laboratório Municipal de Referência Regional - LACEN/Serrinha-Ba) e que residem no mesmo município de Serrinha-Ba, com idade entre 35 e 70 anos, de ambos os sexos, sendo 100 do sexo feminino e 100 do sexo masculino. O município de Serrinha, e uma das cidades que está inserida no semiárido baiano a $173 \mathrm{~km}$ de sua capital Salvador, tem atualmente uma população com cerca de 82.733 habitantes. A atividade econômica gira em torno da agropecuária, indústria e mineração. Os indivíduos convidados já se encontravam no LACEN, para realizarem seus exames de rotina, sendo informados que se aceitassem participar da pesquisa, os exames dos marcadores tumorais biológicos selecionados na investigação, só seriam acrescentando no cadastro, não sendo necessária uma segunda coleta. A inclusão dos marcadores tumorais CA 19.9, o AFP e o CEA, na ficha de cadastro dos indivíduos convidados, só foram feitas, depois de devido esclarecimentos sobre a pesquisa e leitura do Termo de Consentimento Livre e Esclarecido. A coleta de sangue foi realizada pela pesquisadora que foi devidamente treinada e qualificada, e uma vez coletado o sangue as amostras foram armazenadas adequadamente e enviadas para o Laboratório Central de Saúde Publica Professor Gonçalo Moniz (LACEN/Salvador) na cidade de Salvador - Ba - Brasil, para a dosagem da presença dos antígenos dos respectivos marcadores tumorais biológicos (CEA, AFP, CA 19-9).

\section{RESULTADOS E DISCUSSÃO}

Dentre os 200 individuo analisadas verificou-se que 70 exames apresentaram os valores séricos acima da faixa de referência populacional, sendo que a maioria era do sexo feminino com o total de 43, pacientes com resultados alterados, como apresentado na tabela 2, enquanto os pacientes do sexo masculino apresentaram um total de 29 resultados com alterações nos valores séricos, como podem ser observados na tabela 3 , observa-se também que em alguns indivíduos, houve alterações em dois dos exames dosados e outros apresentarão elevação nos três marcadores avaliados.

Tabela 2. Resultados dos exames dos marcadores tumorais, dos indivíduos do sexo feminino que apresentaram valores acima da faixa de referência populacional.

\begin{tabular}{cccc}
\hline $\mathbf{N}^{\circ}$. Indivíduos & CEA & AFP & CA 19.9 \\
\hline $\mathbf{1}$ & 8,8 & 129,3 & 18,0 \\
$\mathbf{2}$ & 2,25 & 32,12 & 2,29 \\
$\mathbf{3}$ & 0,74 & 5,77 & 39,3 \\
$\mathbf{4}$ & 2,08 & 3,51 & 58,8 \\
$\mathbf{5}$ & 32,28 & 8,39 & 8,2 \\
$\mathbf{6}$ & 58,43 & 832,7 & 61,1 \\
$\mathbf{7}$ & 3,54 & 1,62 & 48,6 \\
$\mathbf{8}$ & 2,5 & 2,79 & 42,2 \\
$\mathbf{9}$ & 65,5 & 1123,6 & 38,7 \\
$\mathbf{1 0}$ & 0,63 & 49,49 & 69,3 \\
$\mathbf{1 1}$ & 1,35 & 105,5 & 2,0 \\
$\mathbf{1 2}$ & 18,99 & 96,18 & 9,2 \\
$\mathbf{1 3}$ & 23,0 & 170,1 & 16,87 \\
$\mathbf{1 4}$ & 32,9 & 4,65 & 2,0 \\
$\mathbf{1 5}$ & 6,83 & 82,2 & 12,1 \\
$\mathbf{1 6}$ & 24,06 & 325,25 & 31,0 \\
$\mathbf{1 7}$ & 5,37 & 76,36 & 8,7 \\
& 3,81 & 80,97 & 8,7
\end{tabular}




\begin{tabular}{|c|c|c|c|}
\hline 19 & 71,67 & 6,52 & 14,0 \\
\hline 20 & 6,54 & 38,5 & 10,6 \\
\hline 21 & 7,16 & 53,63 & 14,0 \\
\hline 22 & 25,3 & 45,1 & 56,7 \\
\hline 23 & 32,1 & 18,3 & 63,3 \\
\hline 24 & 7,6 & 38,6 & 72,1 \\
\hline 25 & 48,1 & 63,2 & 12,7 \\
\hline 26 & 104,5 & 225,0 & 11,8 \\
\hline 27 & 1,24 & 85,3 & 9,32 \\
\hline 28 & 2,43 & 73,2 & 18,6 \\
\hline 29 & 1,32 & 55,8 & 17,1 \\
\hline
\end{tabular}

Tabela 3. Resultados dos exames dos marcadores tumorais, dos indivíduos do sexo masculino que apresentaram valores acima da faixa de referência populacional.

\begin{tabular}{cccc}
\hline $\mathbf{N}^{\circ}$. Indivíduos & CEA & AFP & CA 19.9 \\
\hline $\mathbf{1}$ & 58,32 & 22,61 & 215,3 \\
$\mathbf{2}$ & 38,78 & 8,1 & 45,9 \\
$\mathbf{3}$ & 5,93 & 2,06 & 40,06 \\
$\mathbf{4}$ & 2,99 & 27,02 & 3,87 \\
$\mathbf{5}$ & 1,63 & 49,55 & 6,4 \\
$\mathbf{6}$ & 21,8 & 6,8 & 18,7 \\
$\mathbf{7}$ & 3,38 & 5,4 & 49,1 \\
$\mathbf{8}$ & 16,66 & 112,1 & 52,1 \\
$\mathbf{9}$ & 4,84 & 6,1 & 61,4 \\
$\mathbf{1 0}$ & 28,8 & 46,7 & 67,6 \\
$\mathbf{1 1}$ & 22,3 & 55,1 & 72,1 \\
\hline $\mathbf{1 2}$ & 10,6 & 38,6 & 53,4 \\
\hline
\end{tabular}

Com base na figura 1, que mede o percentual de elevação do total dos 200 pacientes analisados, o marcador tumoral biológico que mais apresentou elevação nos seus valores séricos dosados, foi o AFP, já os marcadores CEA e CA 19.9, apresentam um percentual de elevação próximo. Estes resultados apontam para uma diferenciação na elevação do AFP que pode estar associado a sua alta sensibilidade, sendo este marcador tumoral sensível a outras patologias ou situações fisiológicas que irá culminar em sua elevação. Como por exemplo, em pacientes que apresentam quadro de hepatite aguda e crônica como a cirrose, além de gestantes com crianças que apresentam defeitos do tubo neural, podem apresentar elevação discreta ou moderada nos níveis de AFP [15]. Porém em adultos, valores elevados de AFP (acima de $500 \mathrm{ng} / \mathrm{mL}$ ) são observados em apenas três situações: em neoplasias localizadas nos testículos e ovários; tumores com metástases para o fígado (com origem em outros órgãos) e no carcinoma hepatocelular [16].

A diferença e as tendências de alterações nos resultados dos marcadores tumorais entre homens e mulheres podem ser observadas na figura 2 no qual indica o marcador tumoral CA 19.9, com um índice de elevação maior entre os homens e o AFP entre as mulheres. Tais dados podem ser justificados pelo fato de que a população do sexo masculino apresenta uma maior probabilidade de desenvolver o câncer de pâncreas, para o qual o marcador em questão (CA 19.9) é mais suscetível, o desenvolvimento do câncer de pâncreas pode estar relacionada com o tabagismo, idade, gênero, história familiar, diabetes, pancreatite crônica e exposição ocupacional intensa a determinadas substâncias químicas e corantes, o que de acordo com o MS/INCA, os homens são mais expostos a essas situações que as mulheres. Já o AFP, proteína que é sintetizada principalmente pelo fígado fetal, que apresentou seu maior grau de alterações entre a população do sexo feminino, pode ser entendida pelo fato de em mulheres grávidas esses valores tendem a aumentar de acordo com a evolução da gestação [17].

Uma vez identificados os pacientes que apresentarem valores acima da faixa de referência populacional (valores acima do esperado para cada marcador), estes pacientes foram contatados através de telefone solicitado no memento do preenchimento do cadastro e foi sugerido o encaminhamento médico para que os exames pudessem ser melhor avaliados e se diagnosticar possíveis patologias. Os pacientes que concordaram em ser avaliados pelo médico foram encaminhados à secretaria de saúde a fim de fazer o agendamento da consulta. 
Figura 1: Análise do percentual de elevação dos marcadores tumorais biológicos dos 200 pacientes analisados.

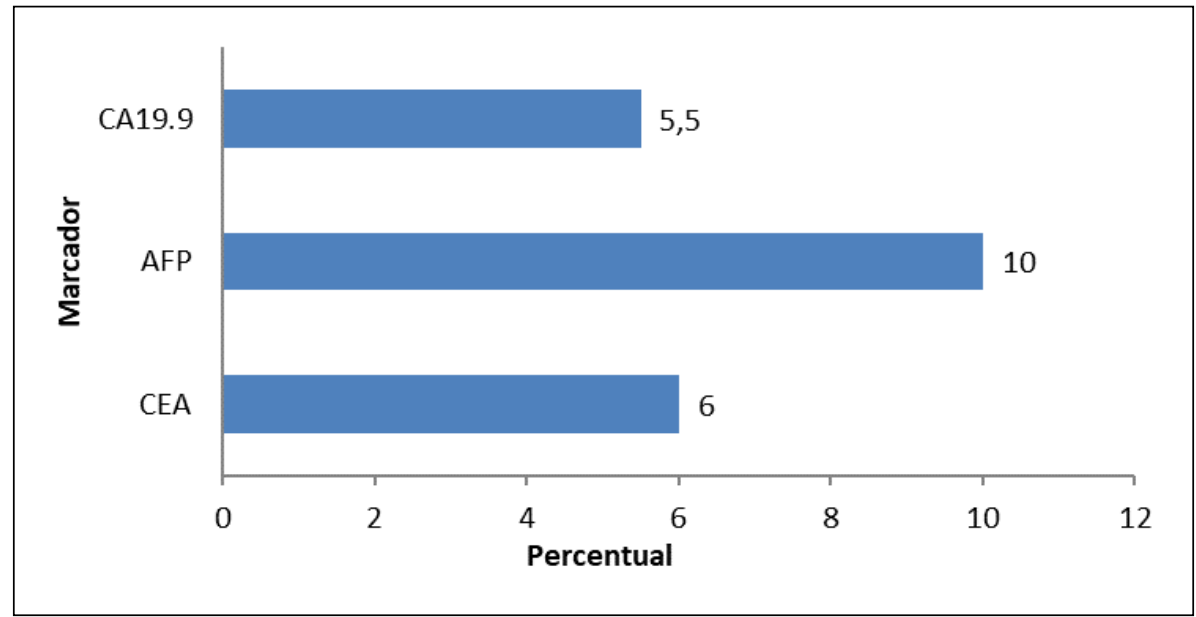

Figura 1: OBS: não existe diferença significativa entre os percentuais dos Marcadores pelo teste para diferença entre proporções $(p>0,05)$.

Figura 2: Análise do percentual de comparação das tendências de alterações dos marcadores tumorais entre os homens e as mulheres investigadas.

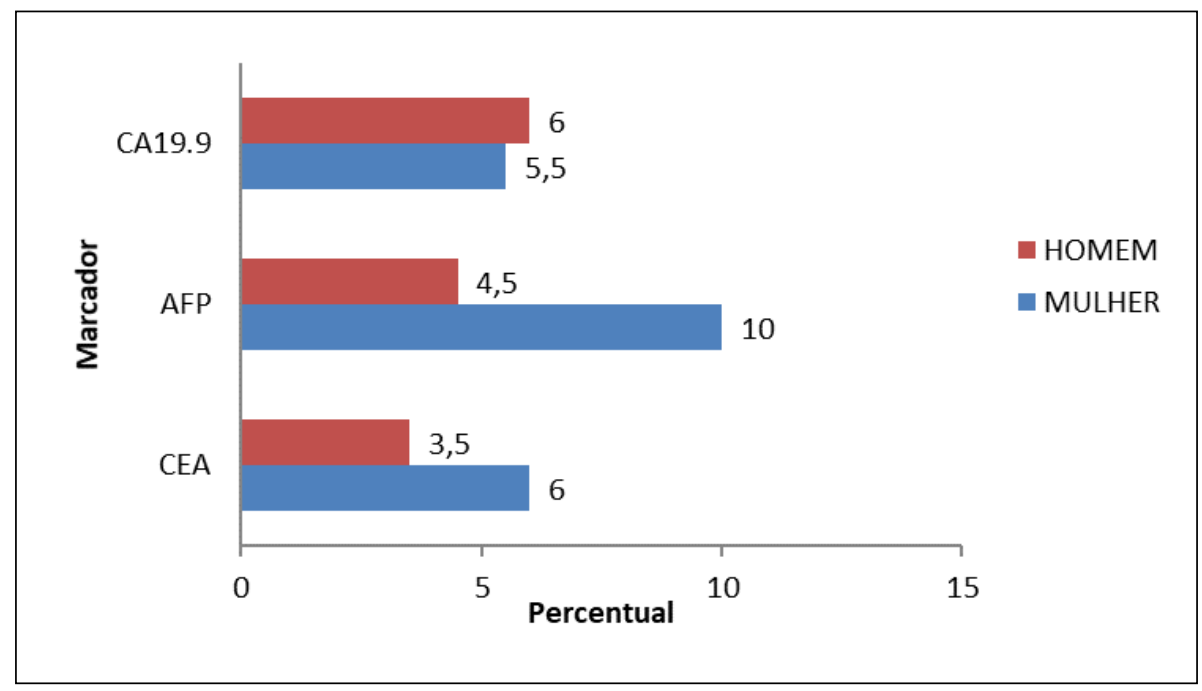

Figura 2: Obs: Resultados significativos para ALFA entre homens e mulheres pelo teste para diferença entre proporções $(p<0,05)$.

\section{CONCLUSÕES}

Uma vez que o câncer fígado e pâncreas encontram-se entre os dez primeiros tipos de câncer com maior incidência, sendo a quinta causa de morte por câncer no Brasil os marcadores tumorais AFP e o CA 19.9 foram os que apresentaram maior percentual de elevação, na população em estudo, demostrando que os dados obtidos seguem o mesmo perfil encontrado na literatura consultada.

Diante do exposto, existe evidência suficiente da importância no uso de marcadores para detecção precoce de câncer, dados estes corroborados pelas observações do INCA para a prevenção do câncer, e particularmente o câncer gastrointestinal, pâncreas e fígado, aos quais os marcadores tumorais biológicos dosados tem maior disponibilidade. Além da prevenção, a realização de exames de rotina e avaliação médicas regulares são de extrema relevância para o acompanhamento no tratamento. O MS/INCA preconiza que medidas como alimentação adequada (rica em fibras, frutas e vegetais), reduzir a quantidade de gorduras na alimentação, bem como manter o peso na medida adequada, diminuir o consumo 
de álcool e não fumar, tudo isso associado à atividade física adequada, podem prevenir de 3 a 4 milhões de novos casos da doença a cada ano no mundo.

Desta forma os resultados obtidos acabam demostrando um perfil nas tendências do risco de desenvolver câncer no município e, portanto, um importante alerta a autoridade local em buscar meios educativos, preventivos e de controle frente ao desenvolvimento dessa doença.

\section{AGRADECIMENTOS}

Ao LACEN (Laboratório Municipal de Referência Regional LACEN: Serrinha-Ba, pela participação direto e consensual na análise e triagem dos usuários da unidade laboratorial de saúde e ao LBC: Laboratório de Biologia Celular (UEFS), onde os professores pesquisadores realizaram a pesquisa.

\section{REFERÊNCIAS}

[1] DeVITA, VT, HELMAN S, and ROSENBERGS SA. Cancer: Principles and Practice of Oncology, 10th ed. Washington, 2014.

[2] TEIXEIRA, L. V., GUERRA, T. A, CONRADO, F. O, TERRA, S. R., GERARDI, D. G. GONZÁLEZ, F.H.D. Evaluation of tumor markers carcinoembryonic antigen, cytokeratin 19 fragment and cancer-associate -antigen 72-4 in neoplastic and nonneoplastic canine effusions differentiation, Arq. Bras. Med. Vet. Zootec., v.66, n.5, p.1311-1316. Rio Grande do Sul. 2014.

[3] TUXEN, M., DOMBERNOWSKY, P. Serum tumour marker CA 125 in monitoring of ovarian cancer during first-line chemotherapy, British Journal of Cancer, 2011.

[4] ALMEIDA, V.L. et al. Câncer e agentes antineoplásicos ciclo-celular específico e ciclo-celular não específico que interagem com o DNA: uma introdução. Química Nova: V. 28, jan/fev. 2005, p. 118-129.

[5] BASSOTTI G, Villanacci. Colonic diverticular disease: abnormalities of neuromuscular function. 2012.

[6] NAKATA B.; OGAWA T.T.; ISHIKAWA T. Serum Cyfra 21.1 is a useful tumor marker for detecting disease relapse and assessing treatment efficacy in breast cancer. $\mathrm{Br} \mathrm{J}$ Cancer, 91:873-878, 2004.

[7] ALVES, V.S. Um modelo de educação em saúde para o Programa de Saúde da Família: pela integralidade da atenção e reorientação do modelo assistencial. Interface - Comunicação, Saúde Educação, Botucatu. 2004.

[8] POIRIER MC, Chemical-induced DNA damage and human cancer risk, Nature Reviews Cancer 4, 630-637. August. 2004.

[9] MINISTÉRIO DA SAÚDE. INSTITUTO NACIONAL DO CÂNCER JOSÉ ALENCAR GOMES SILVA (INCA). Estimativa 2015: Incidência de Câncer no Rio de Janeiro: INCA, 2014.

[10] RIBEIRO, E. M., ARRUDA, A. P. et al. Genética do Câncer Hereditário, Revista Brasileira de Cancerologia, 2009.

[11] MINISTÉRIO DA SAÚDE. INSTITUTO NACIONAL DO
CÂNCER JOSÉ ALENCAR GOMES SILVA (INCA). Estimativa 2016: Incidência de Câncer no Brasil. Rio de Janeiro: INCA, 2015.

[12] LOPES, A. A. et al. Principais genes que participam da formação de tumores, Revista de Biologia e Ciências da Terra, Vol. 2, no 2, 2002.

[13] MARCONI, M. de A.; LAKATOS, E. M. Técnicas de pesquisa In: Fundamentos de Metodologia Científica. Editora Atlas S.A. São Paulo, 2010.

[14] StatSoft, Inc. (2005). STATISTICA (data analysis software system) cersion 7.1: www.statsoft.com

[15] 2010ALMEIDA, J. R. C. et al. Marcadores Tumorais: Revisão de Literatura. Revista Brasileira de Cancerologia: 53(3), 305-316. 2007.

[16] PARDINI, Hermes, Manual de Exames, Belo Horizonte MG. 2013/2014.

[17] WHO \& IARC. GLOBOCAN 2000: Cancer Incidence, Mortality and Prevalence Worldwide. Disponível em: http://wwwdepdb.iarc.fr/globocan/GLOBOframe.htm.

[18] ALVES, SIMONETTI AC, MELO DE LIMA HJ, ANDRADE BARBOSA CE, DE VASCONCELOS SOARES V. Variações dos valores séricos do CEA, bibirrubinas e aminotrasnferases em indivíduos oncológicos. Rev. Bras. An. Clin. 2017.

[19] HOSKOVEC D, VARGA J, KONECNÁ E, ANTOS F. Levels of CEA and Ca 19- 9 in the sera and peritoneal cavity in patients with gastric and pancreatic cancers. Acta Cirúrgica Brasileira, Vol. 27 (6). 2012.

[20] JIAXIN Z, GUANG C, PENG Z, JIAYING Z, XilAKE Li, DA'NAN G, XU CAO, MEI H, HONGBO Du, YONG' an Ye. The threshold of alpha-fetoprotein (AFP) for the diagnosis of hepatocellular carcinoma: A systematic review and metaanalysis. PLOS ONE. 13, 2020.

[21] YANLI He, LIJUAN Z, Glycans and Glycosaminoglycans as Clinical Biomarkers and Therapeutics - Part A. in Progress in Molecular Biology and Translational Science. Brenner's Encyclopedia of Genetics (Second Edition). 2019. Fonte: https:// www.sciencedirect.com/topics/biochemistry-genetics-andmolecular-biology/alpha-fetoprotein

[22] LEE T, GENG TZJ, SHELAT VG. Carbohydrate antigen 19-9 - tumor marker: Past, present, and future. World J Gastrointest Surg 12(12): 468, 2020.

[23] OMER BURCAK BINICIER ZEHRA BETUL PAKOZ. CA 19-9 levels in patients with acute pancreatitis due to gallstone and metabolic/toxic reasons. Rev. Assoc. Med. Bras. 65 (7), 2019. 